\title{
Micromagnetic Simulation of Spin Transfer Torque Magnetization Precession Phase Diagram in a Spin-Valve Nanopillar under External Magnetic Fields
}

\author{
H. B. Huang, ${ }^{1,2}$ X. Q. Ma, ${ }^{1}$ Z. H. Liu, ${ }^{1}$ X. M. Shi, ${ }^{1}$ T. Yue, ${ }^{1}$ Z. H. Xiao, ${ }^{1}$ and L. Q. Chen ${ }^{2}$ \\ ${ }^{1}$ Department of Physics, University of Science and Technology Beijing, Beijing 100083, China \\ ${ }^{2}$ Department of Materials Science and Engineering, The Pennsylvania State University, University Park, PA 16802, USA
}

Correspondence should be addressed to X. Q. Ma, xqma@sas.ustb.edu.cn

Received 29 May 2012; Accepted 21 June 2012

Academic Editors: N. Birge and A. A. Kordyuk

Copyright () 2012 H. B. Huang et al. This is an open access article distributed under the Creative Commons Attribution License, which permits unrestricted use, distribution, and reproduction in any medium, provided the original work is properly cited.

\begin{abstract}
We investigated spin transfer torque magnetization precession in a nanoscale pillar spin-valve under external magnetic fields using micromagnetic simulation. The phase diagram of the magnetization precession is calculated and categorized into four states according to their characteristics. Of the four states, the precessional state has two different modes: steady precession mode and substeady precession mode. The different modes originate from the dynamic balance between the spin transfer torque and the Gilbert damping torque. Furthermore, we reported the behavior of the temporal evolutions of magnetization components in steady precession mode at the condition of the applied magnetic field using the orbit projection method and explaining perfectly the magnetization components evolution behavior. In addition, a result of a nonuniform magnetization distribution is observed in the free layer due to the excitation of non-uniform mode.
\end{abstract}

\section{Introduction}

There has been intensive interest in spintronics for the past two decades [1]. An important example is giant magnetoresistance (GMR), proposed by Fert et al. [2] and Grünberg et al. [3], which has led to commercial products [4]. GMR effect has many potential applications such as magnetic sensor, magnetic read head, data memory and spin transistor, and so forth. Spin-valve [5], as a simple GMR device, also exhibits the switching effect. The merit of spin-valve is that the magnetization reversal can be realized under a small driving magnetic field. Besides being driven by the magnetic field, the magnetization may also be driven by a torque, spin transfer torque (STT), originated from the electron spins in the conducting current. The angular momentums may be transferred from the electrons of the spin-polarized current to the ferromagnet, giving rise to a switching of magnetization or stable precession of magnetizations [6] that leads to the generation of spin waves [7]. This mechanism of STT was initially proposed by Berger et al. [8] and Slonczewski [9] in 1996 and attracted significant interests because of the great potential for direct current-induced spintronic devices [10-12]. The role of STT in magnetization switching has been verified by numerous experiments in spin-valve nanopillars [13-15], magnetic nanowires [16, 17], point contact geometry [18-20], and magnetic tunnel junctions [21-24]. The most attractive application of currentinduced magnetization switching is magnetic random-access memory (MRAM), which has the advantages of nonvolatile, high addressing speed, low-energy consumption, and avoidance of cross writing. Another important application is the current-induced spin transfer nanooscillators (STNO), in which the magnetization precesses, causing a spin wave in the microwave range. In previous researches, it was shown that the application of magnetic field affects the magnitudes of switching field and switching time [25-28], opening new perspectives for information technology. Previous studies have shown that there are different magnetization types in the switching process depending on the magnetic field and the current [14]. Therefore, the understanding of magnetization precession and its mechanism under the external magnetic fields is very important for designing functional devices. 
Many attempts have been made to increase the output power of STNOs; for example, an effective way to increase output power is magnetic-tunnel-junction- (MTJ-) based STNO with a larger linewidth [29-32]. Compared with MTJ-STNO, there are several advantages that STNO with full metal GMR structure have, such as narrow oscillation linewidth, low device resistance, and good impedance matching. Therefore, it is very important to understand the magnetization precession state under the external magnetic field in the conventional 3d-FM-based GMR structure. The phase diagram of magnetization precession under in-plane external field has been studied by several authors [14, 26, 33-35]. Kiselev et al. [36] showed that several different precessional modes exist, that is, small angle precession, large angle precession, and out-of-plane precession. Gmitra et al. [37] presented the phase diagram of magnetic field versus current density, illustrating that precessional state (PS) occurs at small current density and external magnetic fields. Although there have been a number of works on the phase diagram, the detailed analysis of the phase diagram is lacking. Xiao et al. [38] demonstrated that there were several precessional states in the magnetization reversal, that is, large-amplitude, in-plane, and out-of-plane precession from theory. In addition, they also presented the formation of phase diagram from the balance of STT and Gilbert damping torque. However, few published papers showed the detailed discussion of in-plane precession in the phase diagram.

In this paper, we present a systematic study on magnetization precession under spin-polarized currents and magnetic fields using micromagnetic simulation. The magnetization precession is grouped into several types according to their characteristics, which are unchanging state (UN), precessional state (PS), multidomain state (MS), and bistable state (BS). Among them, PS under magnetic field has two precession modes. Furthermore, the orbit projection method is applied to explain the magnetization component oscillation, and the snapshots of magnetization distribution show nonuniform magnetization precession. The magnetization precession phase diagram is helpful for understanding the magnetization precession mode and affecting the oscillation linewidth during the design of spin transfer nanooscillators.

\section{Theoretical Model}

The magnetization precession of a nanoscale pillar structure is investigated under the influence of spin-polarized currents and magnetic fields by micromagnetic simulations based on the Landau-Lifshitz-Gilbert (LLG) equation incorporating STT effect. The nanopillar structure is schematically shown in Figure 1. The thicknesses of each layer are Co $(2 \mathrm{~nm}) / \mathrm{Cu}$ $(4 \mathrm{~nm}) / \mathrm{Co}(10 \mathrm{~nm})$ from top to bottom, and the size of the nanopillar is $16 \times 64 \times 16 \mathrm{~nm}^{3}$. The two Co layers are separated by a thin $\mathrm{Cu}$ layer; the top Co layer is the free layer whose magnetization precession is triggered by a spinpolarized current. The bottom Co layer is the pinned layer with its magnetization vector $\mathbf{P}$ fixed in the direction along the positive $x$-axis. The initial magnetization vector $\mathbf{M}$ of the layer is along the negative or positive $x$-axis. The middle $\mathrm{Cu}$ layer is a space layer whose function is to avoid the exchange

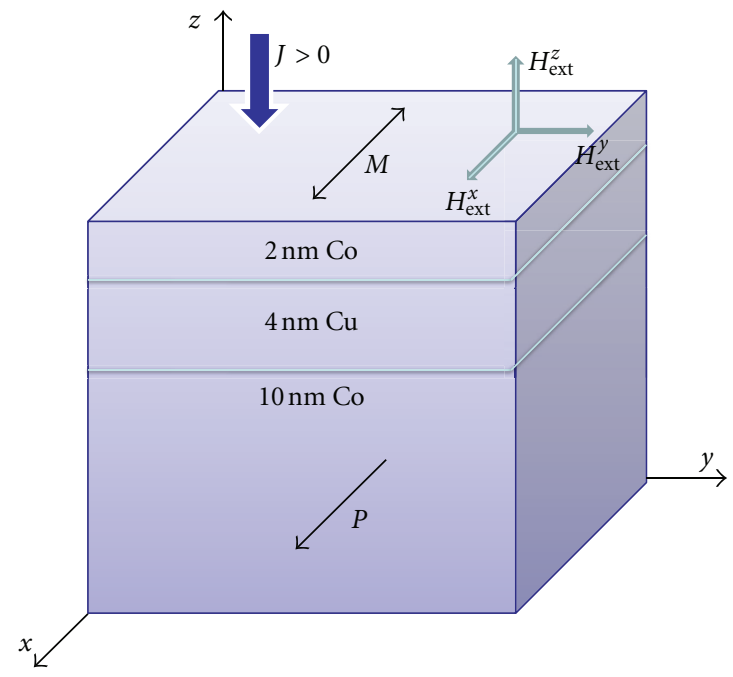

FIgURE 1: Model geometry definition of $\mathrm{Co} / \mathrm{Cu} / \mathrm{Co}$ nanopillar in Cartesian coordinates.

coupling between the two Co layers. The thickness of the spacer layer $(4 \mathrm{~nm})$ is much smaller than the spin diffusion length to conserve the spin momentum. The positive current is defined as the current flow from the free layer to the pinned layer.

The dynamics could be described by the temporal evolution of the magnetization $\mathbf{M}$, and $\mathbf{M}$ obeys the generalized Landau-Lifshitz-Gilbert-Slonczewski (LLGS) equation:

$$
\begin{aligned}
\frac{d \mathbf{M}}{d t}= & -\gamma^{\prime} \mathbf{M} \times \mathbf{H}_{\mathrm{eff}}-\frac{\alpha \gamma^{\prime}}{M_{s}} \mathbf{M} \times\left(\mathbf{M} \times \mathbf{H}_{\mathrm{eff}}\right) \\
& -\frac{2 \mu_{B} J}{\left(1+\alpha^{2}\right) e d M_{s}^{3}} g(\mathbf{M}, \mathbf{P}) \mathbf{M} \times(\mathbf{M}, \mathbf{P}) \\
& +\frac{2 \mu_{B} \alpha J}{\left(1+\alpha^{2}\right) e d M_{s}^{2}} g(\mathbf{M}, \mathbf{P})(\mathbf{M}, \mathbf{P}) .
\end{aligned}
$$

The first term on the right-hand side of (1) is a conventional magnetic torque with gyromagnetic ratio $\gamma$, where $\gamma^{\prime}=\gamma /\left(1+\alpha^{2}\right)$. This torque is driven by an effective field,

$$
\mathbf{H}_{\mathrm{eff}}=-\frac{1}{\mu_{0}} \frac{\delta E}{\delta \mathbf{M}} .
$$

The effective field of the LLGS includes the anisotropy, demagnetization, external, and the exchange fields, namely, $\mathbf{H}_{\text {eff }}=\mathbf{H}_{\text {exch }}+\mathbf{H}_{\text {ani }}+\mathbf{H}_{\mathrm{ms}}+\mathbf{H}_{\text {ext }}$. Taking account of exchange energy, anisotropy energy, magnetostatic energy, and Zeeman energy, the total energy $E$ is given by

$$
E=E_{\mathrm{ani}}+E_{\mathrm{exch}}+E_{\mathrm{ms}}+E_{\mathrm{ext}} \text {. }
$$

The anisotropy energy of $\mathrm{Co}$ in a $\mathrm{Co} / \mathrm{Cu} / \mathrm{Co}$ nanopillar is

$$
E_{\text {ani }}=\int\left(K_{1}\left[1-m_{s}^{2}(r)\right]+K_{2}\left[1-m_{s}^{2}(r)\right]^{2}\right) d^{3} r,
$$

where $K_{1}$ and $K_{2}$ are the anisotropy constants, $m_{s}(r)$ is the unit magnetization vector at each grid, $m_{s}(r)=M_{s}(r) / M_{s}$, and the easy axis is along the $x$-axis. Considering the fact that 


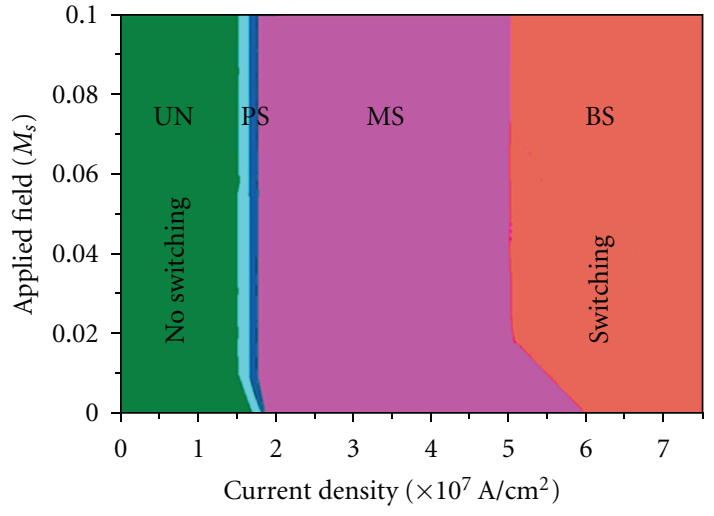

(a)

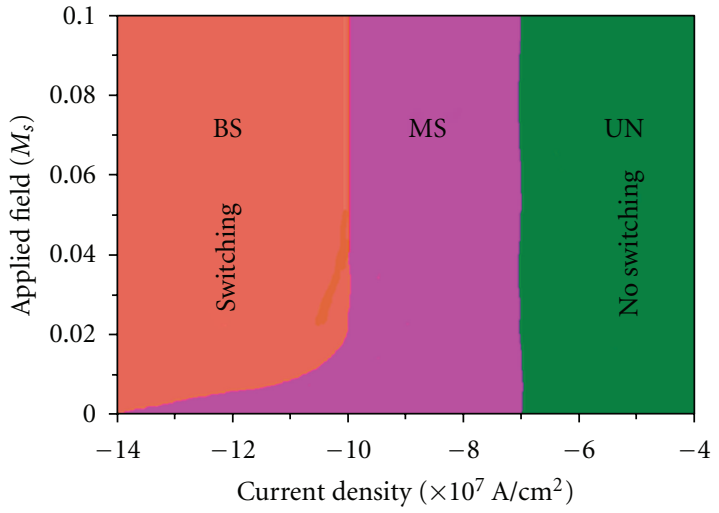

(b)

FIGURE 2: Phase diagram of magnetization precession under perpendicular magnetic fields (a) for a positive current and (b) for a negative current.

$K_{1}$ is far greater than $K_{2}$, we ignore the second term in the simulations.

The exchange energy is

$$
E_{\text {exch }}=\int\left(\frac{A}{M_{s}^{2}}|\nabla M(r)|^{2}\right) d^{3} r
$$

where $A$ is the exchange stiffness constant.

The magnetostatic energy can be presented as a sum of energies of interacting magnetic dipoles as follows:

$$
\begin{aligned}
E_{\mathrm{ms}}=\frac{1}{2} \iint\left\{M_{i}(r)\left[\frac{\delta_{i j}}{\left|r-r^{\prime}\right|^{3}}-\frac{3\left(r_{i}-r_{i}^{\prime}\right)\left(r_{j}-r_{j}^{\prime}\right)}{\left|r-r^{\prime}\right|^{5}}\right]\right. \\
\left.\times M_{j}(r)\right\} d^{3} r d^{3} r^{\prime} .
\end{aligned}
$$

We utilize the fast Fourier transform (FFT) technique for obtaining the magnetostatic energy as it involves double integrals in real space.

The Zeeman energy is

$$
E_{\mathrm{ext}}=-\mu_{0} \int\left[H_{\mathrm{ext}} \cdot M(r)\right] d^{3} r,
$$

where $H_{\text {ext }}$ is the externally applied magnetic field.

The second term in (1) is the "Gilbert damping" which takes into account energy dissipation, such as coupling to lattice vibrations [39] and spin-flip scattering [40]. This is used by most practitioners, although there is an active debate whether this form of the damping is correct [41-43].

The last two terms on the right side of (1) describe STT which tends to drag the magnetization away from its initial state to its final state and drive the magnetization precession around the effective field. The scalar function is given by [9]

$$
g(\mathbf{M}, \mathbf{P})=\left[-4+\frac{(1+\eta)^{3}\left(3+\mathbf{M} \cdot \mathbf{P} / M_{s}^{2}\right)}{4 \eta^{3 / 2}}\right]^{-1} .
$$

where $\mathbf{H}_{\text {STT }}$ is the corresponding effective field given by

$$
\mathbf{H}_{\mathrm{STT}}=\frac{2 \mu_{B} J g(\mathbf{M}, \mathbf{P}) \mathbf{M} \times \mathbf{P}}{\left(\gamma e d M_{s}^{3}\right)},
$$

where $\mu_{B}, J, d, e$, and $M_{s}$, are the Bohr magneton, current density, thickness of the free layer, electron charge, and saturation magnetization, respectively.

Analysis and micromagnetic calculations based on this model show that magnetization reversal in the layer requires an initial deviation from strictly parallel or antiparallel alignment. If $\mathbf{M}$ is parallel or antiparallel to $\mathbf{P}\left(\theta=0^{\circ}\right.$ or $\theta=$ $180^{\circ}$ ), STT is zero as $\mathbf{M} \times \mathbf{P}=\mathbf{0}$. To initiate the simulation, a small angle deviation from the parallel or antiparallel configuration between $\mathbf{M}$ and $\mathbf{P}$ was assigned. The classical Oersted field scales as $1 / r$ generated by conduction electrons, where $r$ is the lateral size of the free layer, while STT scales as $1 / r^{2}$. Therefore, for small sizes like a nanopillar with the cross-section $64 \times 64 \mathrm{~nm}^{2}$, STT dominates over the classical Oersted field. We ignore the Oersted field and thermal activation in this work for studying a "minimal" model of the spin torque-driven magnetization precession. In our simulations, we assumed $\theta=0.1^{\circ}$ for parallel and $\theta=179.9^{\circ}$ for antiparallel configurations at the beginning. The parameters of Co are adopted from the values at helium temperature $(4.2 \mathrm{~K})[26]: \eta=0.35, M_{s}=1.446 \times 10^{6} \mathrm{~A} / \mathrm{m}$, $\gamma=2.3245 \times 10^{5} \mathrm{~m} /(\mathrm{A} \cdot \mathrm{s}), K_{1}=6.86 \times 10^{5} \mathrm{~J} / \mathrm{m}^{3}$, $\alpha=0.01, A=2.0 \times 10^{-11} \mathrm{~J} / \mathrm{m}$, and $d=2 \mathrm{~nm}$. The dynamics of magnetization is investigated by numerically solving the time-dependent LLGS equation using the GaussSeidel projection method $[44,45]$ with a constant time step $\Delta t=14.875 \mathrm{fs}$. Simulation performed with shorter time step gave the same results. The free layer is discrete in computational cells of $2 \times 2 \times 2 \mathrm{~nm}^{3}$ [46].

\section{Results}

Figure 2 shows the phase diagram of magnetization precession under perpendicular magnetic fields along $z$-axis direction. Each precessional state corresponds to a certain current density range under external field. The case of positive current results in magnetic switching from antiparallel state to parallel state, like the situation without magnetic field [26]. The magnetization process can be grouped into four types: unchanging state (UN), precessional state (PS), 


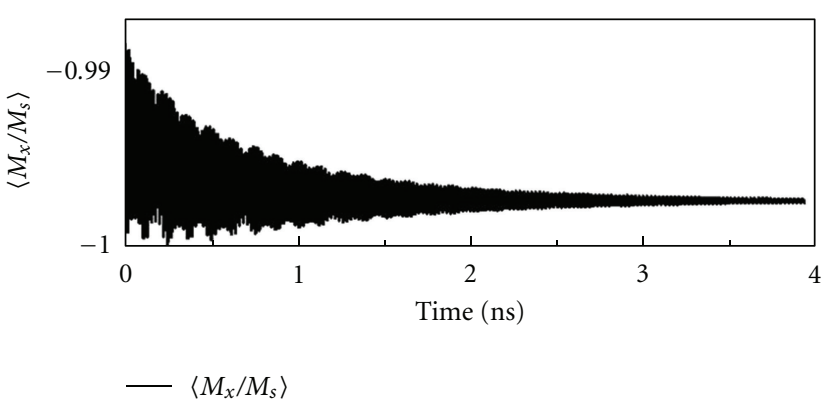

(a)

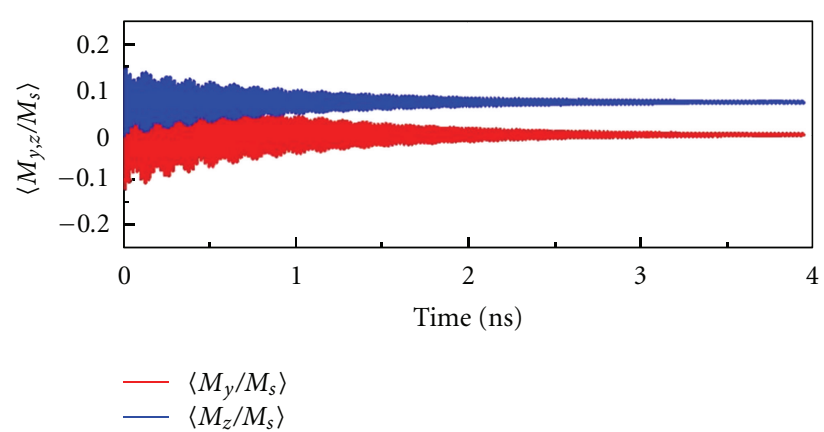

(b)

Figure 3: The temporal evolution of the magnetization components under $J=1.00 \times 10^{7} \mathrm{~A} / \mathrm{cm}^{2}$.

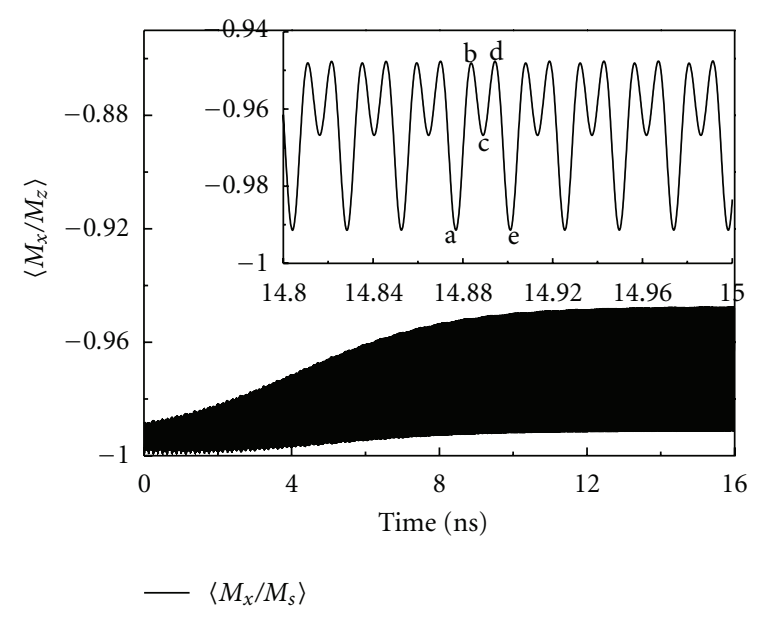

(a)

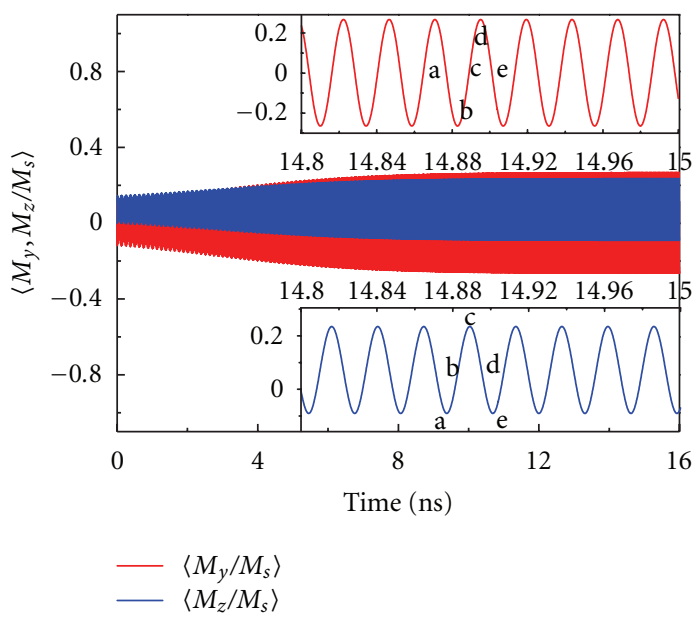

(b)

Figure 4: The temporal evolutions of the magnetization components under $J=1.60 \times 10^{7} \mathrm{~A} / \mathrm{cm}^{2}$. The insets are the zooms of time span from $14.80 \mathrm{~ns}$ to $15.00 \mathrm{~ns}$. The five points (a, b, c, d, and e) correspond to the times of $14.877 \mathrm{~ns}, 14.883 \mathrm{~ns}, 14.890 \mathrm{~ns}, 14.893 \mathrm{~ns}$, and $14.899 \mathrm{~ns}$, respectively.

multidomain state (MS), and bistable state (BS). In UN, the magnetization keeps in the initial antiparallel state. The magnetizations in PS precess around the effective field with steady motion in the film plane. In MS, the magnetization lies in the multidomain state. Only in BS, the magnetization can be switched from the initial antiparallel state to the final parallel state. However, different from the situation of the uniform state in PS under zero field [26], when the external magnetic field is applied, two precession modes were found in this state: steady and substeady precession modes.

A negative current favors antiparallel state, resulting in the magnetic reversal from the initial parallel to final antiparallel state, as shown in Figure 2(b). Different from the positive current case, only three types of states were observed in the reverse process with PS missing.

To illustrate the feature and mechanism of magnetization precession under perpendicular magnetic fields, we take $H_{\mathrm{ext}}^{z}=0.10 M_{s}(1.82 \mathrm{KOe})$ as an example. In this condition, if the current density varies in the range of $0 \leq J<$ $1.50 \times 10^{7} \mathrm{~A} / \mathrm{cm}^{2}$, the magnetization dynamics lies in the US. In this state, STT energy is less than the Gilbert energy dissipation. Any finite fluctuation is damped down, and the magnetization keeps in the initial state. Figures 3(a) and 3 (b) show that the temporal evolutions of magnetization components are heavily damped under $J=1.00 \times 10^{7} \mathrm{~A} / \mathrm{cm}^{2}$.

In the range of $1.50 \times 10^{7} \mathrm{~A} / \mathrm{cm}^{2} \leq J<1.95 \times$ $10^{7} \mathrm{~A} / \mathrm{cm}^{2}$, the magnetization dynamics lies in the PS, where the Gilbert energy dissipation equals the STT energy input. PS corresponds to the in-plane precession (IPP) in the film plane; the out-of-plane precession (OPP) is not found in our micromagnetic simulations, which means the transition from the precessional state to multidomain state occurs directly in the presence of magnetic fields. The absence of OPP was identified as a consequence of the underestimation of the exchange constant and the role of the initial selfmagnetostatic field, which was demonstrated by Jaromirska et al. [47]. The two modes in the PS, that is, steady precession mode, and substeady precession mode, are in the ranges of $1.50 \times 10^{7} \mathrm{~A} / \mathrm{cm}^{2} \leq J<1.65 \times 10^{7} \mathrm{~A} / \mathrm{cm}^{2}$ and $1.65 \times$ $10^{7} \mathrm{~A} / \mathrm{cm}^{2} \leq J \leq 1.95 \times 10^{7} \mathrm{~A} / \mathrm{cm}^{2}$, respectively.

Figure 4 shows the temporal evolution of the magnetization components in the steady precession mode. The 


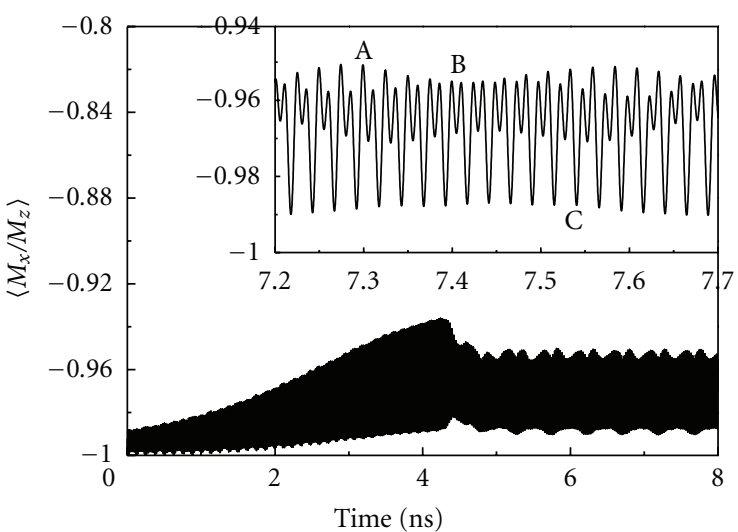

$-\left\langle M_{x} / M_{s}\right\rangle$

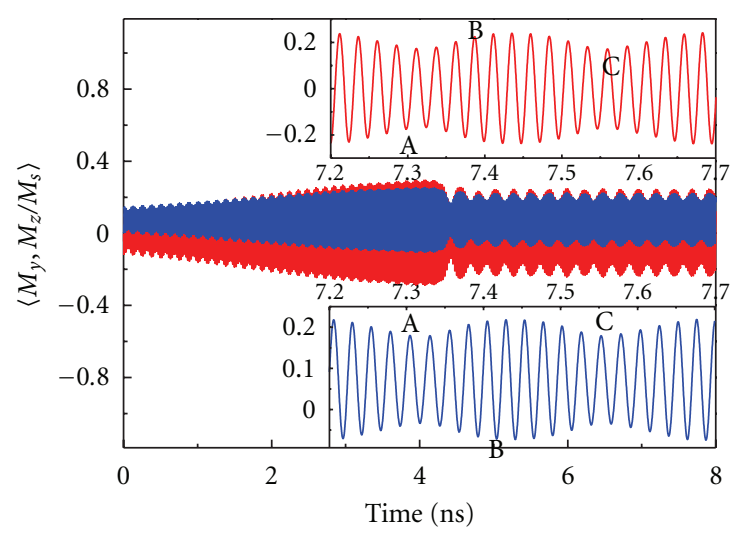

$-\left\langle M_{y} / M_{s}\right\rangle$

(a)

(b)

Figure 5: The temporal evolution of the magnetization components under $J=1.70 \times 10^{7} \mathrm{~A} / \mathrm{cm}^{2}$. The insets show the "beat" behavior of magnetization in the zoom of $0.5 \mathrm{~ns}$. The three points (A, B, and C) correspond to the time of $7.299 \mathrm{~ns}, 7.410 \mathrm{~ns}$, and $7.540 \mathrm{~ns}$, respectively.

temporal evolution of $\left\langle M_{x} / M_{s}\right\rangle$ shows two stages: initial and stable stages. In the initial stage, the Gilbert damping torque is smaller than the STT, therefore, the amplitude of $\left\langle M_{x} / M_{s}\right\rangle$ increases with the time evolution. Then the Gilbert energy dissipation equals the STT energy input in the stable stage; the magnetic moments precess around the effective field, with oscillations at constant frequency and amplitude. Different from the precession without magnetic field, the temporal evolution of the magnetization component $\left\langle M_{x} / M_{s}\right\rangle$ has a "sag" in the oscillation crest at a perpendicular magnetic field. Although this phenomenon had also been reported by Siracusano et al. [48] and Horley et al. [49, 50], the explanation is not found. We shall give a systematic explanation of this phenomenon later.

In the range of $1.65 \times 10^{7} \mathrm{~A} / \mathrm{cm}^{2} \leq J \leq 1.70 \times$ $10^{7} \mathrm{~A} / \mathrm{cm}^{2}$, the magnetization lies in the substeady precession mode. Figure 5 depicts the temporal evolutions of the magnetization components under $J=1.70 \times 10^{7} \mathrm{~A} / \mathrm{cm}^{2}$. The amplitude is modulated by a low-frequency sinusoidal contour, showing the feature of "beat" phenomenon. The beat frequency equals to $4.03 \mathrm{GHz}$ and this phenomenon does not appear in the case of zero applied fields. Though Finocchio et al. [51] and Berkov and Gorn [52] reported this mode, the mechanism is not presented. We will show the discussion of the mechanism.

Figure 6 shows the temporal evolution of the magnetization components. In the range of $1.95 \times 10^{7} \mathrm{~A} / \mathrm{cm}^{2} \leq$ $J<5.5 \times 10^{7} \mathrm{~A} / \mathrm{cm}^{2}$, the magnetization dynamics lies in the MS, which corresponds to the region "W" described in [14]. The mechanism is similar to that of PS. The differences are that the free layer is not a monodomain and the amplitudes of magnetization oscillation are not constant. This multidomains evolution process could be explained by the large current input energy. The energy per unit time pumped into the nanopillar by the current is so large, that the formation of magnetic excitations with the wavelength much shorter than the element size becomes possible, leading to the formation of multidomains.

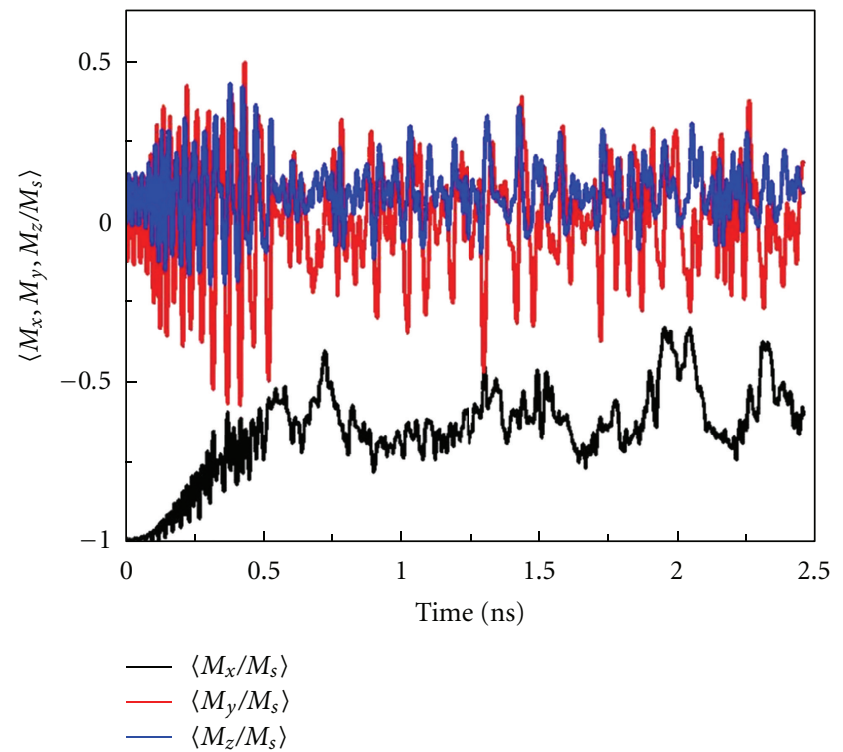

FIGURE 6: The temporal evolution of the magnetization components under $J=5.0 \times 10^{7} \mathrm{~A} / \mathrm{cm}^{2}$.

When $-\infty<J \leq-10.0 \times 10^{7} \mathrm{~A} / \mathrm{cm}^{2}$ and $5.5 \times$ $10^{7} \mathrm{~A} / \mathrm{cm}^{2} \leq J<\infty$, the magnetization can be switched by the STT. As shown in Figure 7, the magnetization dynamics lies in the BS. In this state, the magnetization can be switched back and forth between two stable states: parallel or antiparallel configurations. The threshold current densities are $J_{c}=$ $5.5 \times 10^{7} \mathrm{~A} / \mathrm{cm}^{2}$ from antiparallel state to parallel state and $J_{-c}=-10.0 \times 10^{7} \mathrm{~A} / \mathrm{cm}^{2}$ from parallel state to antiparallel state. The magnetization switching is accompanied by drastic magnetization oscillation. In fact, as soon as the current is applied, the magnetization oscillation occurs until the final static state is reached. Comparing with the critical current density of the zero fields, the threshold switching current under perpendicular magnetic fields is lower. This result can explain that the applied field changes the direction of 


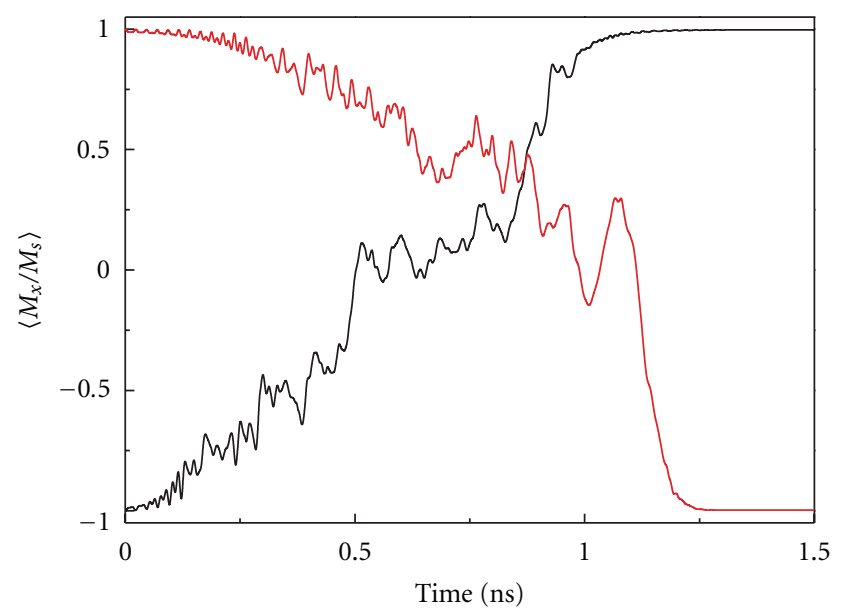

- Positive current

- Negative current

Figure 7: The temporal evolution of the magnetization components under $9.0 \times 10^{7} \mathrm{~A} / \mathrm{cm}^{2}$ (black curve) and $-16.0 \times 10^{7} \mathrm{~A} / \mathrm{cm}^{2}$ (red curve).

effective field and stimulates the magnetization precession around it.

\section{Discussion}

To understand magnetization precession phase diagram of spin-valve nanopillar under perpendicular external magnetic fields, one needs to study the characteristics of STT and Gilbert damping torque. The balance between them determines the final states of the system.

STT can be described by the Slonczewski's model, which takes into account the interface spin-flip scattering effect. STT versus $\theta$ is described by (9), and Gilbert damping torque is proportional to the function $\sin \theta[53,54]$. They are plotted in Figure 8, where the current density is $9.0 \times$ $10^{7} \mathrm{~A} / \mathrm{cm}^{2}$, and the perpendicular external magnetic field of $0.10 M_{s}$ is applied. STT reaches its maximum at $\theta=127.17^{\circ}$, which leads to easier reversal from the antiparallel to the parallel state than that of the reverse process. Thus $\left|J_{c}\right|(5.5 \times$ $\left.10^{7} \mathrm{~A} / \mathrm{cm}^{2}\right)$ is smaller than $\left|J_{-c}\right|\left(\left|-10.0 \times 10^{7} \mathrm{~A} / \mathrm{cm}^{2}\right|\right)$.

As shown in Figure 9, in UN, the total torque is negative in all ranges of $\theta$ since STT is less than the Gilbert damping torque in this state. Therefore, any finite oscillation is damped and the magnetization keeps in the initial state.

There are two curves of total torque for PS in Figure 9, corresponding to two precession modes. Stable precession mode occurs at angles where the total torque changes from positive to negative, which means the Gilbert energy dissipation equals STT energy input in this case. In the steady precession mode, the total torque is always equal to zero with $\theta$ in the range from $178^{\circ}$ to $179^{\circ}$. The magnetization trajectory is precessing around the effective field, showing a limiting cycle of saddle-shaped orbit which is plotted in Figure 10(a). The color curves stand for the projections on the planes Oxy (red), Oxz (green), and Oyz (blue), respectively. The simulated magnetization dynamics has a

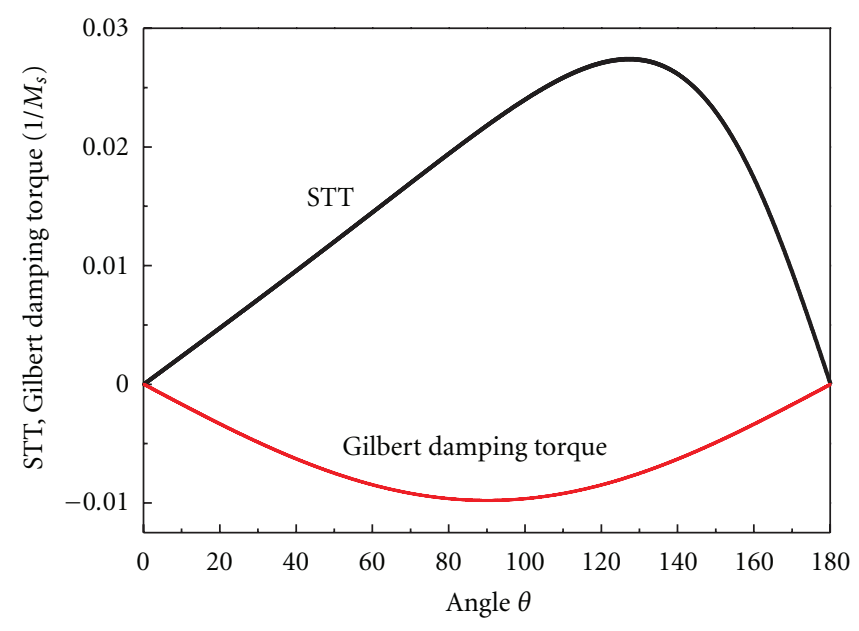

Figure 8: The relationships of STT and Gilbert damping torque versus $\theta$ of Slonczewski's model.

steady-state character, as can be observed from the temporal evolutions of magnetization components in Figure 4. With the increase of current density, the temporal evolutions of magnetization components lie in substeady precession mode, and the modulation oscillation can be observed in Figure 5. The total torque equals to zero at the range of $166^{\circ} \leq$ $\theta \leq 179^{\circ}$, and the magnetization trajectory precesses with the effective field in this range from the limiting cycle to a band of cycles as shown in Figure 10(b). The quasiperiodic magnetization trajectory filling a bent torus was also shown in $[46,47]$, but we give the explanation of this mode formation. The explanation of the "beat" phenomenon is that since the range of $\theta$ corresponding to the balance between STT and the Gilbert damping torque increases from $178^{\circ} \leq \theta \leq 179^{\circ}$ to $166^{\circ} \leq \theta \leq 179^{\circ}$ with the increase of current density, that is, the range of the precession angle is enlarged, therefore, the transition of the trajectory from the limiting cycle to the band of cycles occurs. In addition, there is no PS in the case of negative current, since the total torque is negative at small $\theta$. This explains the absence of PS in the case of negative current density, as shown in magnetic dynamics phase diagram of Figure 2.

In MS, the total torque becomes positive at the range of $70^{\circ} \leq \theta \leq 179^{\circ}$. It is impossible for the magnetization dynamics to have a steady precessional state at $\theta=70^{\circ}$ because micromagnetic simulation divides the pillar into many cell grids and the magnetization in each domain will precess around the local effective field. It is different from the macrospin model which describes the magnetic particle as a macroscopic magnetic moment $[47,52,53]$.

In BS, STT increases with $\theta$ and eventually destabilizes the initial antiparallel state. When the total torque becomes positive in all ranges of $\theta$, the system abruptly switches to the parallel state. In this state, STT is always bigger than the Gilbert damping torque in the whole switching process so that the total torque may drive the magnetization switching. In addition, the balance between STT and Gilbert damping torque for the negative current has similar behavior except in PS. 


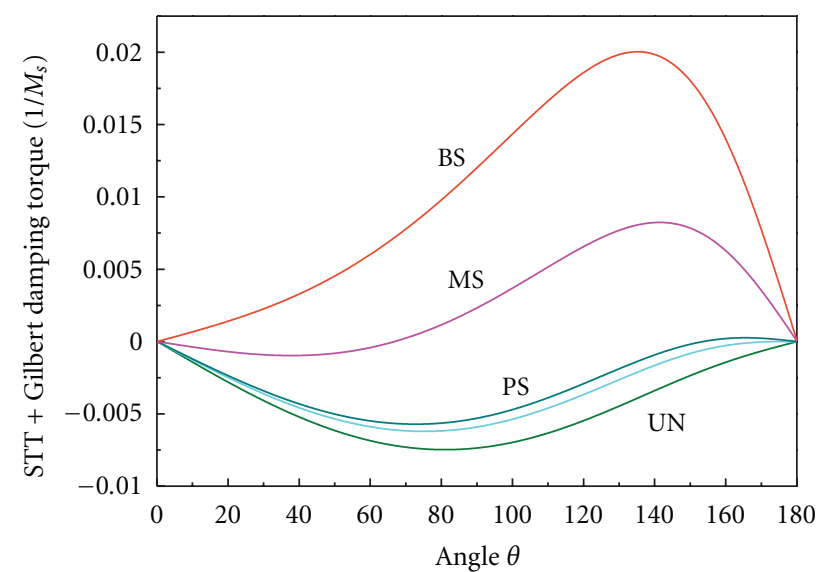

FIGURE 9: The total of STT and Gilbert damping torque versus $\theta$ at different current densities, each color curve stands for the total torque in the different current densities.

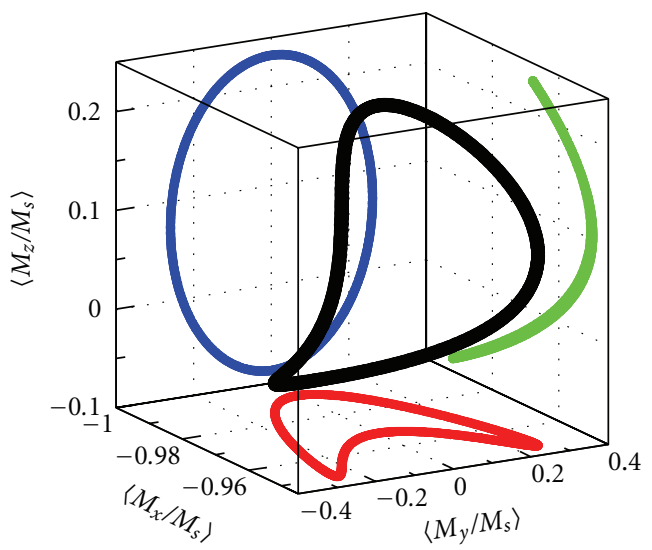

(a)

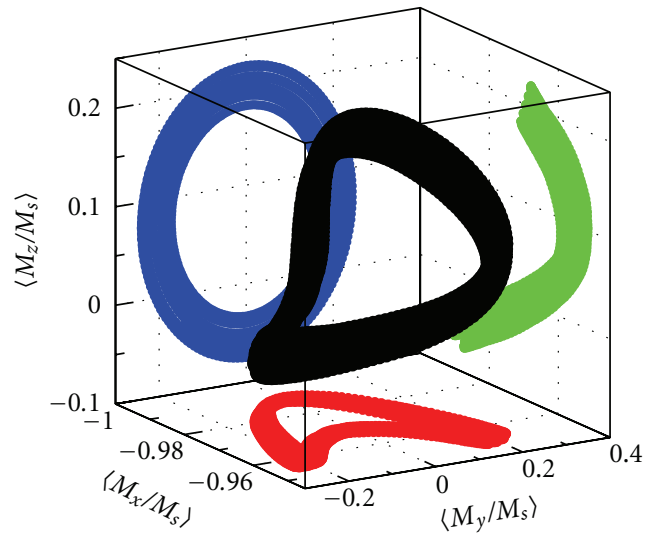

(b)

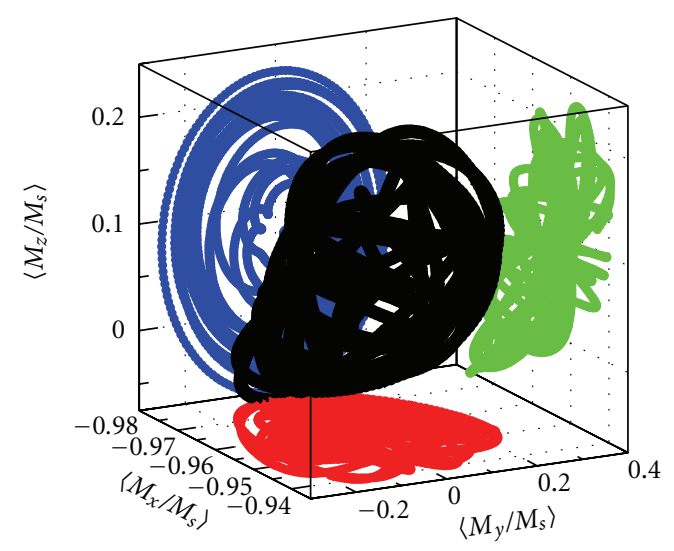

(c)

Figure 10: The trajectories and projections on the planes $O x y$ (red), $O x z$ (green), and $O y z$ (blue): (a) steady precession mode, (b) substeady precession mode, and (c) multidomain precession state.

We show the sketch of magnetization precession trajectories in Figure 11(a) for explaining the "sag" in the oscillation crest of Figure 4 . The magnetization precesses around the effective field $H_{\text {eff }}$ along the saddle-shaped orbit [14]. The temporal evolution of three components $\left\langle m_{x}\right\rangle,\left\langle m_{y}\right\rangle$, and $\left\langle m_{z}\right\rangle$ of magnetization $\langle\mathbf{m}\rangle\left(\mathbf{m}=\mathbf{M} / M_{s}\right)$ may be obtained by projecting the magnetization trajectory onto three axes. For example, the magnetization trajectory under the $x$-axis magnetic field can be projected to the plane $O x y$ and then to the $x$-axis to get the $x$-component of magnetization $\left\langle m_{x}\right\rangle$, as 


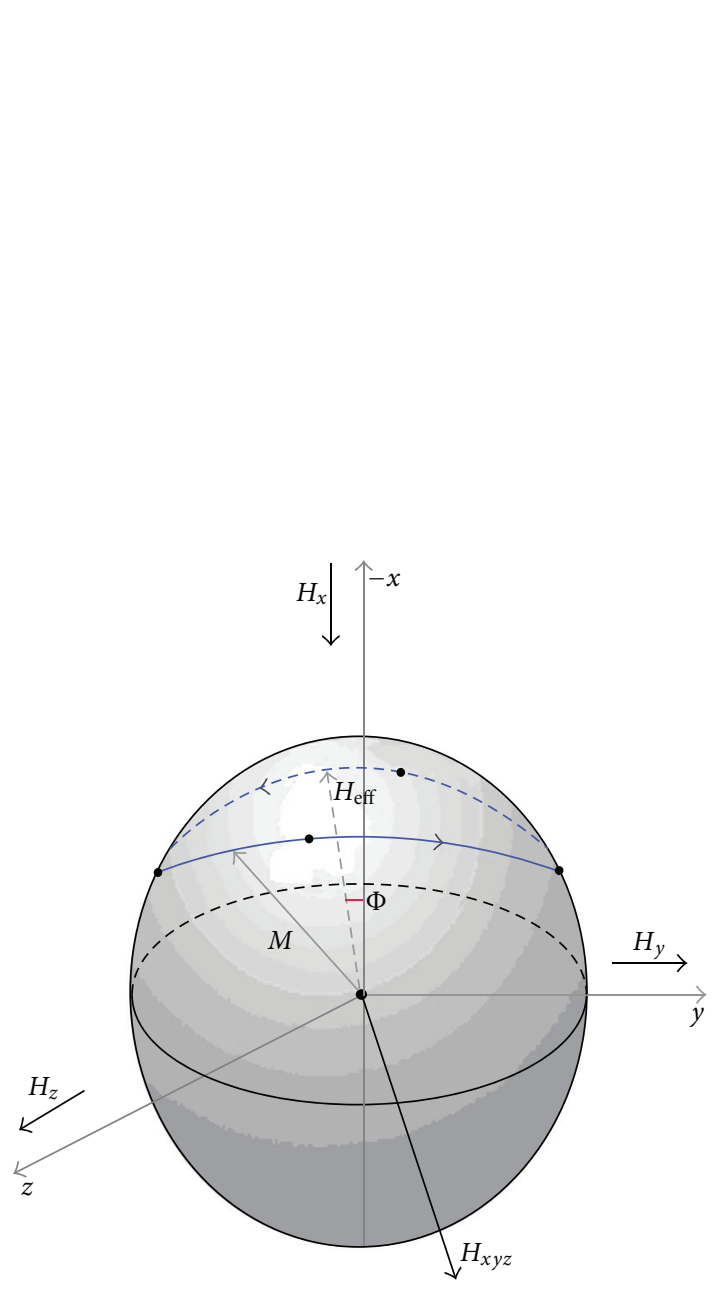

(a)

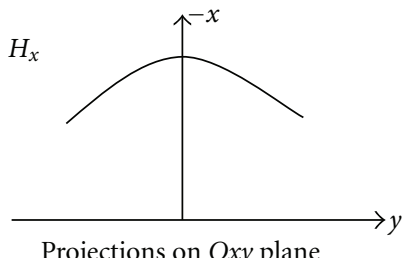

Projections on Oxy plane

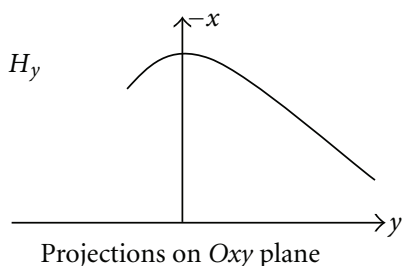

Projections on Oxy plane

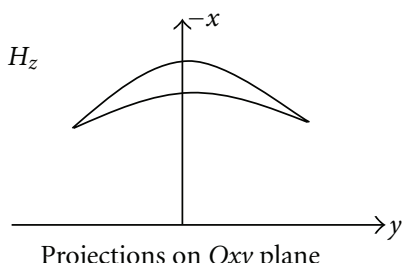

Projections on Oxy plane

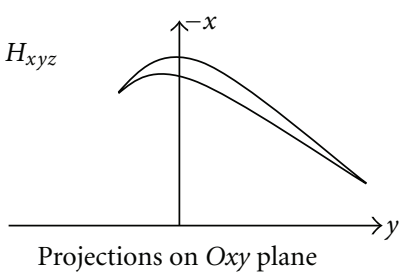

(b)
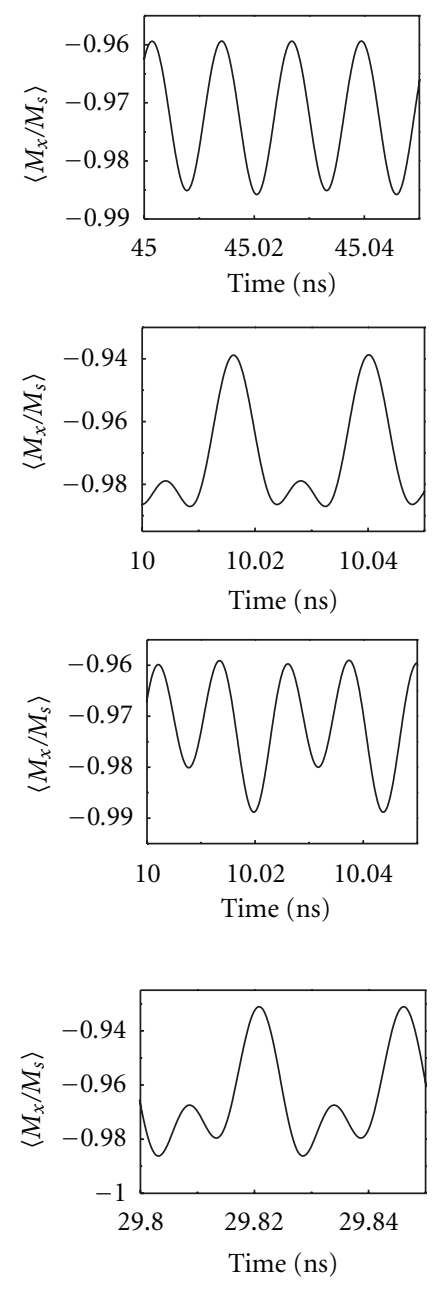

Figure 11: (a) Sketch of magnetizationtrajectories, (b) projections on the plane Oxy under different external magnetic fields (left) and temporal evolutions of the average magnetizations $\left\langle m_{x}\right\rangle$ (right).

shown in Figure 11(b). There is a curve with two overlapping minor arcs symmetric to the $x$-axis. When the magnetization is precessing around the $x$-axis, the $\left\langle m_{x}\right\rangle$ component moves back and forth twice during one complete precession cycle, and hence the oscillation frequency of $\left\langle m_{x}\right\rangle$ is approximately twice as large as that for $\langle\mathbf{m}\rangle$ oscillation. The projection under the $y$-axis field is a curve asymmetrical to the $x$-axis and inclining towards $+y$-axis. Thus, the temporal evolution of $\left\langle m_{x}\right\rangle$ has a "salient" in the trough, which results from the asymmetrical projection to the $x$-axis on the plane $O x y$. The projection under the $z$-axis field is a loop with two nonoverlapping minor arcs symmetrical to the $x$-axis, which leads to the result of magnetization oscillation having a "sag" in the crest. For the field along $x y z$-axis, the oscillation has two maximums and two minimums in one cycle of precession, since the projection is a loop asymmetrical to the $x$-axis and inclining towards $+y$-axis.

Furthermore, the "sag" in the crest will disappear as the magnetic field increases from zero to $0.30 M_{s z}\left(H_{z}=\right.$ $\left.0.30 M_{s}\right)$, as shown in Figure 12. In the zero field, the projection on the plane $O x y$ is a curve with two overlapping minor arcs. When the $\langle\mathbf{m}\rangle$ precesses around the effective field for one cycle, the $\left\langle m_{x}\right\rangle$ has two oscillations. There is no "sag" in the crest in this case. But the applied field increases to $0.05 M_{s z}$, there are "sags" in the crests of the temporal evolution of magnetization component $\left\langle m_{x}\right\rangle$, because of the projection of a loop with two nonoverlapping minor arcs. As the applied field increases to $0.20 M_{s z}$, the "sag" in the crest becomes smaller than that for the field of $0.05 M_{s z}$. When the applied field increases to $0.30 M_{s}$, the projection on the plane Oxy becomes a loop with a straight line and an arc from two nonoverlapping minor arcs and the "sag" disappears. Similar behavior can be found in the fields along $y$-axis and $x y z$-axis.

Figure 13 shows the temporal evolution of $\left\langle m_{x}\right\rangle$, trajectory, and snapshots of the magnetization distribution under $H_{y}=0.04 M_{s}$ and $J=1.60 \times 10^{7} \mathrm{~A} / \mathrm{cm}^{2}$. The $\left\langle m_{x}\right\rangle$ has a maximum -0.930 at the point a, a second maximum -0.973 at the point $b$, and two minimums -0.983 at the points $\mathrm{b}$ and $\mathrm{d}$. The five snapshots of magnetization distributions correspond to the five points in the temporal evolution of magnetization. The colors represent the magnitude of the magnetization component $\left\langle m_{z}\right\rangle(-0.3 \sim 0.3)$, while the 


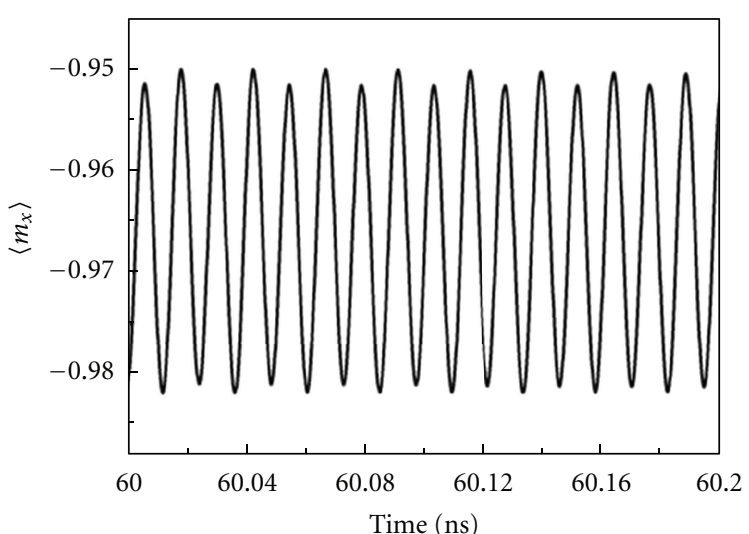

— Zero field

(a)

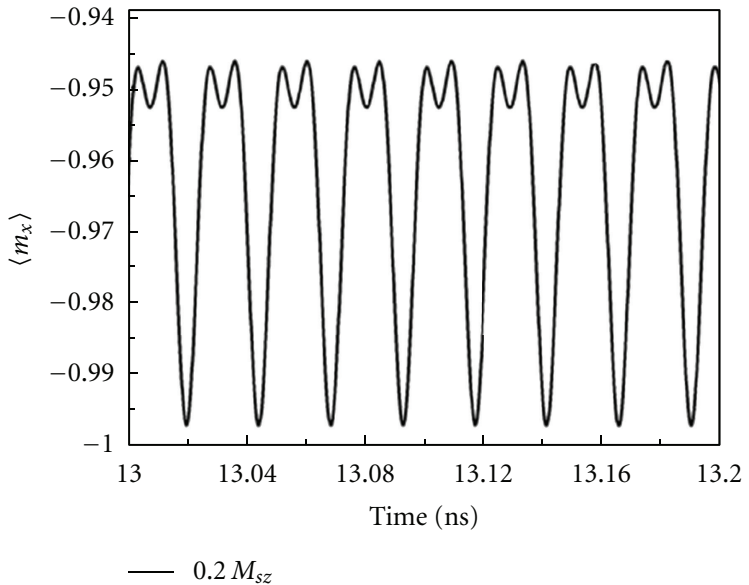

(c)

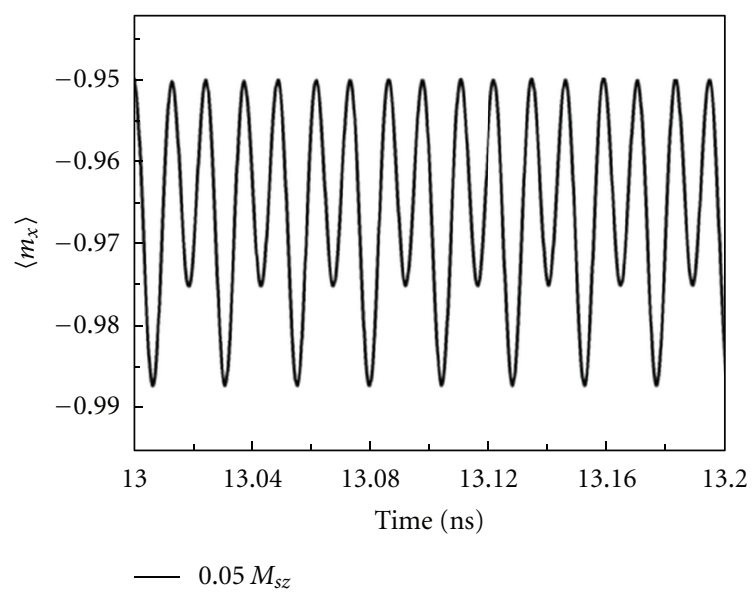

(b)

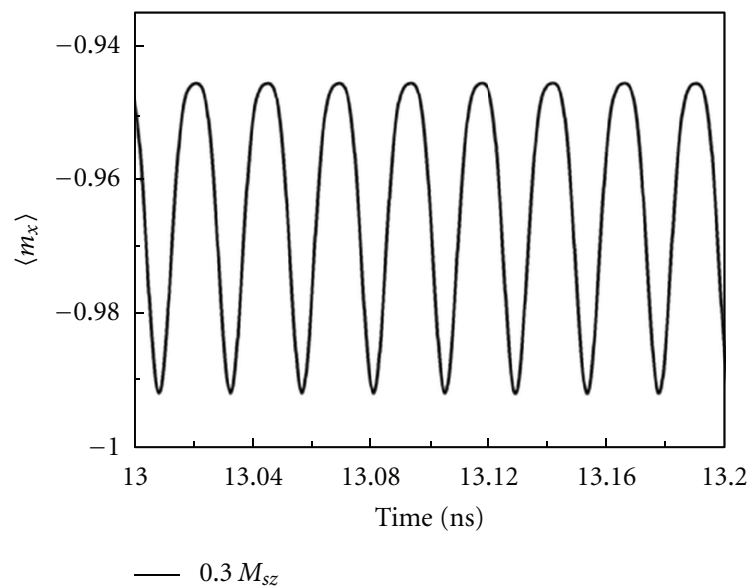

(d)

Figure 12: The temporal evolutions of magnetization components under four different magnetic fields along the $z$-axis.

arrows indicate the magnetization projection on the plane Oxy, through which the magnetization components $\left\langle m_{x}\right\rangle$ and $\left\langle m_{y}\right\rangle$ were shown. The magnetization almost entirely lies in the plane $O x y$ at the point a, where $\left\langle m_{z}\right\rangle$ is nearly zero. At the point $\mathrm{b}$, the average magnetization component $\left\langle m_{z}\right\rangle$ is about -0.2 . The magnetization is first excited at the boundaries of the structure via the nucleation process because of spatially nonuniform local demagnetization fields [33] and becomes broader and broader with time, showing nonuniform magnetization precession. In the point $c$, the magnetization is also on the plane Oxy. However, the arrows rotate toward a different direction. The average magnetization component $\left\langle m_{z}\right\rangle$ is 0.2 at the point $\mathrm{d}$. At the end of the period, the oscillation returns to its original state.

\section{Conclusions}

Based on our micromagnetic simulation of spin transfer torque magnetization precession under magnetic fields in $\mathrm{Co} / \mathrm{Cu} / \mathrm{Co}$ nanopillars, the magnetization process can be grouped into four types: unchanging state (UN), precessional state (PS), multidomain state (MS), and bistable state (BS). Of the four states, PS could be further divided into two modes: steady precession mode and substeady precession mode. Balance of the spin transfer torque and Gilbert damping torque can lead to the three modes; the magnetization dynamics depends on the total torque of STT and Gilbert damping torque. Only in the case of positive current, the PS state can present. In addition, the temporal evolutions of magnetization components in steady precession mode are explained by the method of the orbit projection, and the "sag" in the crest will disappear as the magnetic field increases from zero to $0.30 M_{s z}$. Furthermore, the magnetization distributions together with its trajectory and temporal evolution are shown to elucidate the nonuniform magnetization precession.

\section{Acknowledgments}

This work was sponsored by the National Science Foundation of China (11174030), by the US National Science Foundation under Grant no. DMR-1006541 (Chen), and in part by the China Scholarship Council. The computer simulations were carried out on the LION and Cyberstar clusters at The Pennsylvania State University. 


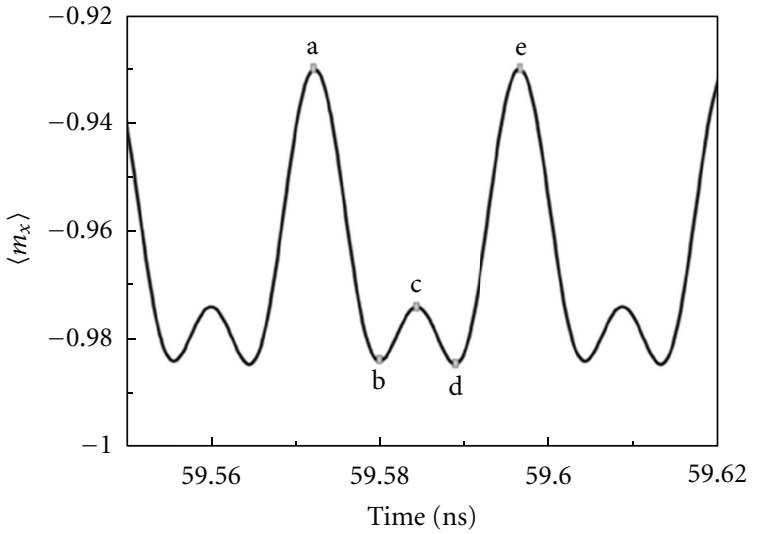

(A)

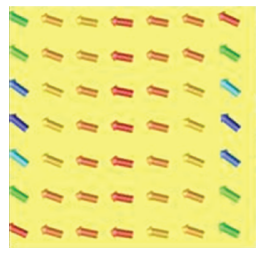

(a)

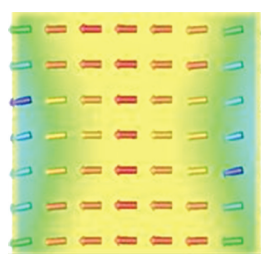

(b)

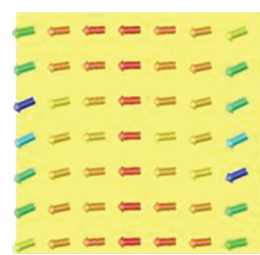

(c)

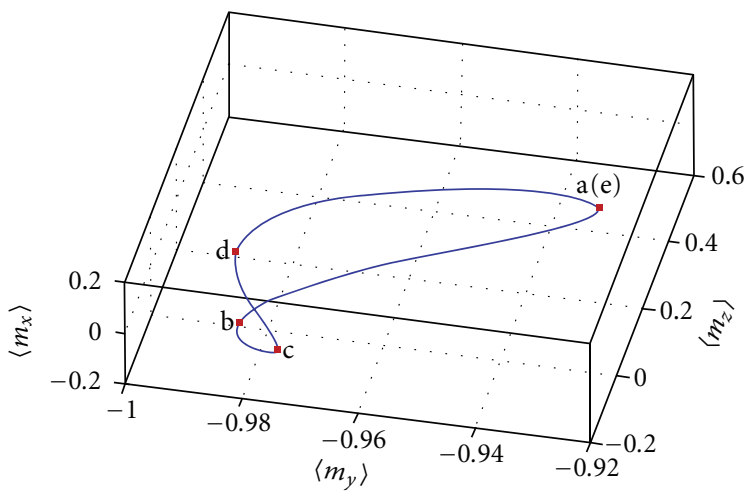

(B)

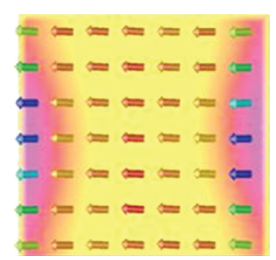

(d)

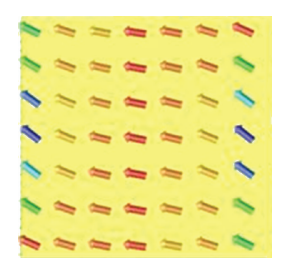

(e)

(C)

Figure 13: (a) The temporal evolution of $\left\langle m_{x}\right\rangle$ in the zoom of $59.55 \sim 59.62 \mathrm{~ns}$ under $J=1.60 \times 10^{7} \mathrm{~A} / \mathrm{cm}^{2}$ and $H_{y}=0.04 M_{s}$. (b) The magnetization precessional trajectory. (c) Micromagnetic simulation result of the snapshots of magnetization distribution. The colors represent the magnitude of the magnetization component $\left\langle m_{z}\right\rangle$ (pink positive, cyan negative).

\section{References}

[1] I. Žutić, J. Fabian, and S. D. Sarma, "Spintronics: fundamentals and applications," Reviews of Modern Physics, vol. 76, no. 2, pp. 323-410, 2004.

[2] M. N. Baibich, J. M. Broto, A. Fert et al., "Giant magnetoresistance of (001)Fe/(001)Cr magnetic superlattices," Physical Review Letters, vol. 61, no. 21, pp. 2472-2475, 1988.

[3] P. Grünberg, R. Schreiber, Y. Pang, M. B. Brodsky, and H. Sowers, "Layered magnetic structures: evidence for antiferromagnetic coupling of Fe layers across Cr interlayers," Physical Review Letters, vol. 57, no. 19, pp. 2442-2445, 1986.

[4] S. S. P. Parkin, N. More, and K. P. Roche, "Oscillations in exchange coupling and magnetoresistance in metallic superlattice structures: $\mathrm{Co} / \mathrm{Ru}, \mathrm{Co} / \mathrm{Cr}$, and Fe/Cr," Physical Review Letters, vol. 64, no. 19, pp. 2304-2307, 1990.

[5] B. Dieny, V. S. Speriosu, S. S. P. Parkin, B. A. Gurney, D. R. Wilhoit, and D. Mauri, "Giant magnetoresistive in soft ferromagnetic multilayers," Physical Review B, vol. 43, no. 1, pp. 1297-1300, 1991.

[6] M. Tsoi, A. G. M. Jansen, J. Bass et al., "Excitation of a magnetic multilayer by an electric current," Physical Review Letters, vol. 80, no. 19, pp. 4281-4284, 1998.

[7] E. B. Myers, D. C. Ralph, J. A. Katine, R. N. Louie, and R. A. Buhrman, "Current-induced switching of domains in magnetic multilayer devices," Science, vol. 285, no. 5429, pp. 867-870, 1999.

[8] B. Fisher, L. Patlagan, and G. M. Reisner, "Transport properties of $\mathrm{L}_{1-x} \mathrm{Sr}_{x} \mathrm{MnO}_{3}(L=\mathrm{Pr}, \mathrm{Nd} ; 1 / 4 \leq \mathrm{x} \leq 1 / 2)$," Physical Review B, vol. 54, pp. 9359-9364, 1996.
[9] J. C. Slonczewski, "Current-driven excitation of magnetic multilayers," Journal of Magnetism and Magnetic Materials, vol. 159, no. 1-2, pp. L1-L7, 1996.

[10] D. C. Ralph and M. D. Stiles, "Spin transfer torques," Journal of Magnetism and Magnetic Materials, vol. 320, pp. 1190-1216, 2008.

[11] G. Tatara, H. Kohno, and J. Shibata, "Microscopic approach to current-driven domain wall dynamics," Physics Reports, vol. 468, no. 6, pp. 213-301, 2008.

[12] J. Z. Sun and D. C. Ralph, "Magnetoresistance and spintransfer torque in magnetic tunnel junctions," Journal of Magnetism and Magnetic Materials, vol. 320, no. 7, pp. 12271237, 2008.

[13] J. Z. Sun, D. J. Monsma, D. W. Abraham, M. J. Rooks, and R. H. Koch, "Batch-fabricated spin-injection magnetic switches," Applied Physics Letters, vol. 81, no. 12, pp. 2202-2204, 2002.

[14] S. I. Klselev, J. C. Sankey, I. N. Krivorotov et al., "Microwave oscillations of a nanomagnet driven by a spin-polarized current," Nature, vol. 425, no. 6956, pp. 380-383, 2003.

[15] S. Urazhdin, N. O. Birge, W. P. Pratt, and J. Bass, "Currentdriven magnetic excitations in permalloy-based multilayer nanopillars," Physical Review Letters, vol. 91, no. 14, Article ID 146803, 4 pages, 2003.

[16] J. E. Wegrowe, X. Hoffer, P. Guittienne et al., "Spin-polarized current induced magnetization switch: is the modulus of the magnetic layer conserved? (invited)," Journal of Applied Physics, vol. 91, no. 10, p. 6806, 2002.

[17] D. Kelly, J.-E. Wegrowe, T.-K. Truong, X. Hoffer, and J.P. Ansermet, "Spin-polarized current-induced magnetization reversal in single nanowires," Physical Review B , vol. 68, no. 13, Article ID 134425, 13 pages, 2003. 
[18] M. Tsol, A. G. M. Jansen, J. Bass, W.-C. Chlang, V. Tsol, and P. Wyder, "Generation and detection of phase-coherent currentdriven magnons in magnetic multilayers," Nature, vol. 406, no. 6791, pp. 46-48, 2000.

[19] W. H. Rippard, M. R. Pufall, S. Kaka, S. E. Russek, and T. J. Silva, "Direct-Current Induced Dynamics in $\mathrm{Co}_{90} \mathrm{Fe}_{10} / \mathrm{Ni}$ ${ }_{80} \mathrm{Fe}_{20}$ Point Contacts," Physical Review Letters, vol. 92, no. 2, pp. 272011-272014, 2004.

[20] T. Y. Chen, Y. Ji, C. L. Chien, and M. D. Stiles, "Currentdriven switching in a single exchange-biased ferromagnetic layer," Physical Review Letters, vol. 93, no. 2, Article ID 026601, 4 pages, 2004.

[21] J. Z. Sun, "Current-driven magnetic switching in manganite trilayer junctions," Journal of Magnetism and Magnetic Materials, vol. 202, no. 1, pp. 157-162, 1999.

[22] Y. Liu, Z. Zhang, P. P. Freitas, and J. L. Martins, "Currentinduced magnetization switching in magnetic tunnel junctions," Applied Physics Letters, vol. 82, no. 17, pp. 2871-2873, 2003.

[23] Y. Huai, M. Pakala, Z. Diao, and Y. Ding, "Spin transfer switching current reduction in magnetic tunnel junction based dual spin filter structures," Applied Physics Letters, vol. 87, no. 22, Article ID 222510, 3 pages, 2005.

[24] D. H. Lee and S. H. Lim, "Increase of temperature due to Joule heating during current-induced magnetization switching of an MgO-based magnetic tunnel junction," Applied Physics Letters, vol. 92, no. 23, Article ID 233502, 3 pages, 2008.

[25] H. Huang, X. Ma, T. Yue, Z. Xiao, S. Shi, and L. Chen, "Magnetization switching modes in nanopillar spin valve under the external field," Science China: Physics, Mechanics and Astronomy, vol. 54, no. 7, pp. 1227-1234, 2011.

[26] X. Q. Ma, Z. H. Xiao, P. P. Wu, J. X. Zhang, S. Q. Shi, and L. Q. Chen, "Current-induced magnetization dynamics in $\mathrm{CoCuCo}$ nanopillars," Journal of Applied Physics, vol. 103, no. 7, Article ID 07B111, 2008.

[27] F. B. Mancoff, R. W. Dave, N. D. Rizzo, T. C. Eschrich, B. N. Engel, and S. Tehrani, "Angular dependence of spintransfer switching in a magnetic nanostructure," Applied Physics Letters, vol. 83, no. 8, pp. 1596-1598, 2003.

[28] P. Weinberger, A. Vernes, B. L. Györffy, and L. Szunyogh, "Noncollinear magnetic structures: a possible cause for current-induced switching," Physical Review B, vol. 70, no. 9, Article ID 094401, 13 pages, 2004.

[29] A. M. Deac, A. Fukushima, H. Kubota et al., "Bias-driven high-power microwave emission from $\mathrm{MgO}$-based tunnel magnetoresistance devices," Nature Physics, vol. 4, no. 10, pp. 803-809, 2008.

[30] D. Houssameddine, S. H. Florez, J. A. Katine et al., "Spin transfer induced coherent microwave emission with large power from nanoscale $\mathrm{MgO}$ tunnel junctions," Applied Physics Letters, vol. 93, no. 2, Article ID 022505, 2008.

[31] Z. M. Zeng, P. Upadhyaya, P. Khalili Amiri et al., "Enhancement of microwave emission in magnetic tunnel junction oscillators through in-plane field orientation," Applied Physics Letters, vol. 99, no. 3, Article ID 032503, 2011.

[32] K. Mizushima, T. Nagasawa, K. Kudo, Y. Saito, and R. Sato, "Decrease of nonlinearity and linewidth narrowing in spintransfer oscillators under the external field applied near the hard axis," Applied Physics Letters, vol. 94, no. 15, Article ID 152501, 2009.

[33] K. J. Lee, A. Deac, O. Redon, J. P. Nozières, and B. Dieny, "Excitations of incoherent spin-waves due to spin-transfer torque," Nature Materials, vol. 3, no. 12, pp. 877-881, 2004.
[34] A. Deac, K. J. Lee, Y. Liu et al., "Spin transfer effects in exchange-biased spin-valves for current-perpendicular-toplane magnetoresistive heads," Journal of Magnetism and Magnetic Materials, vol. 290-291, pp. 42-47, 2005.

[35] L. G. Pereira, O. Boulle, M. M. Sanchez, V. Cros, F. Petroff, and A. Fert, "Current-driven differential resistance phase diagram in nanopillars of NiFe/Cu/NiFe," Physica B, vol. 384, no. 1-2, pp. 33-35, 2006.

[36] S. I. Kiselev, J. C. Sankey, I. N. Krivorotov et al., "Currentinduced nanomagnet dynamics for magnetic fields perpendicular to the sample plane," Physical Review Letters, vol. 93, no. 3, Article ID 036601, 4 pages, 2004.

[37] M. Gmitra and J. Barnaś, "Current-driven destabilization of both collinear configurations in asymmetric spin valves," Physical Review Letters, vol. 96, no. 20, Article ID 207205, 2006.

[38] J. Xiao, A. Zangwill, and M. D. Stiles, "Macrospin models of spin transfer dynamics," Physical Review B, vol. 72, no. 1, Article ID 014446, 13 pages, 2005.

[39] H. Suhl, "Theory of the magnetic damping constant," IEEE Transactions on Magnetics, vol. 34, no. 4, part 1, pp. 18341838, 1998.

[40] V. Kambersky, "On the Landau-Lifshitz relaxation in ferromagnetic metals," Canadian Journal of Physics, vol. 48, no. 24, pp. 2906-2911, 1970.

[41] V. L. Safonov, "Tensor form of magnetization damping," Journal of Applied Physics, vol. 91, no. 10, p. 8653, 2002.

[42] A. Rebei, M. Simionato, and G. J. Parker, "Correlation functions of the magnetization in thin films," Physical Review B, vol. 69, no. 13, Article ID 134412, pp. 134412-10, 2004.

[43] S. Zhang and S. S.-L. Zhang, "Generalization of the LandauLifshitz-Gilbert equation for conducting ferromagnets," Physical Review Letters, vol. 102, no. 8, Article ID 086601, 2009.

[44] X. P. Wang, C. J. García-Cervera, and E. Weinan, "A Gaussseidel projection method for micromagnetics simulations," Journal of Computational Physics, vol. 171, no. 1, pp. 357-372, 2001.

[45] J. X. Zhang and L. Q. Chen, "Phase-field microelasticity theory and micromagnetic simulations of domain structures in giant magnetostrictive materials," Acta Materialia, vol. 53, no. 9, pp. 2845-2855, 2005.

[46] H. B. Huang, X. Q. Ma, Z. H. Liu et al., "Micromagnetic simulation of spin-transfer switching in a full-Heusler $\mathrm{Co}_{2} \mathrm{FeAl}_{0.5} \mathrm{Si}_{0.5}$ alloy spin-valve nanopillar," Journal of Applied Physics, vol. 110, no. 3, Article ID 033913, 2011.

[47] E. Jaromirska, P. Baláž, L. López Díaz, and J. Barnaś, “Computational study of microwave oscillations in nonstandard spin valves in the diffusive transport limit," Physical Review B, vol. 81, no. 1, Article ID 014408, 2010.

[48] G. Siracusano, G. Finocchio, I. N. Krivorotov, L. Torres, G. Consolo, and B. Azzerboni, "Micromagnetic simulations of persistent oscillatory modes excited by spin-polarized current in nanoscale exchange-biased spin valves," Journal of Applied Physics, vol. 105, no. 7, Article ID 07D107, 2009.

[49] P. P. Horley, V. R. Vieira, P. M. Gorley, V. K. Dugaev, and J. Barnaś, "Current-induced dynamics of a monodomain ferromagnet in an external magnetic field applied in easy magnetic plane: macrospin model," Physical Review B, vol. 77, no. 9, Article ID 094427, 2008.

[50] P. P. Horley, V. R. Vieira, P. M. Gorley, V. K. Dugaev, J. Berakdar, and J. Barna, "Influence of a periodic magnetic field and spin-polarized current on the magnetic dynamics of a monodomain ferromagnet," Physical Review B, vol. 78, no. 5, Article ID 054417, 2008. 
[51] G. Finocchio, V. S. Pribiag, L. Torres, R. A. Buhrman, and B. Azzerboni, "Spin-torque driven magnetic vortex selfoscillations in perpendicular magnetic fields," Applied Physics Letters, vol. 96, no. 10, Article ID 102508, 2010.

[52] D. Berkov and N. Gorn, "Transition from the macrospin to chaotic behavior by a spin-torque driven magnetization precession of a square nanoelement," Physical Review B, vol. 71, no. 5, Article ID 052403, 4 pages, 2005.

[53] D. V. Berkov and N. L. Gorn, "Magnetization precession due to a spin-polarized current in a thin nanoelement: numerical simulation study," Physical Review B, vol. 72, no. 9, Article ID 094401, 2005.

[54] G. Finocchio, G. Siracusano, V. Tiberkevich, I. N. Krivorotov, L. Torres, and B. Azzerboni, "Time-domain study of frequency-power correlation in spin-torque oscillators," Physical Review B, vol. 81, no. 18, Article ID 184411, 2010. 

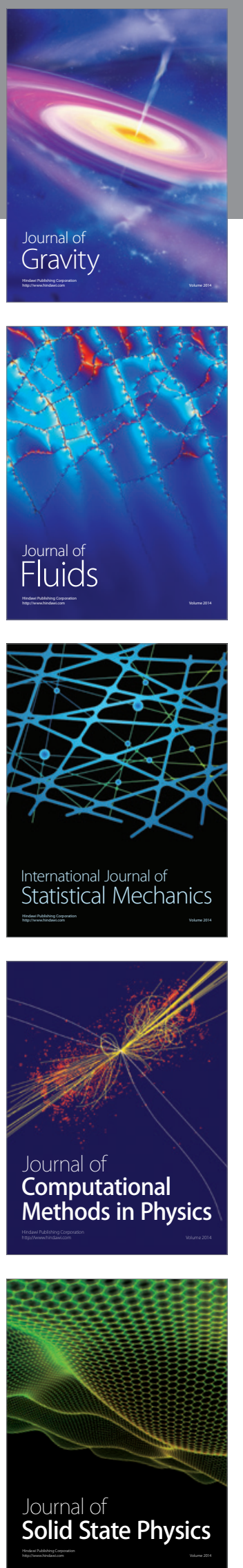
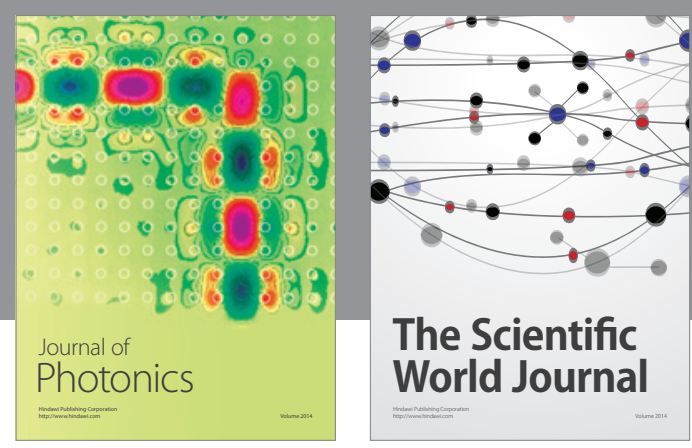

The Scientific World Journal

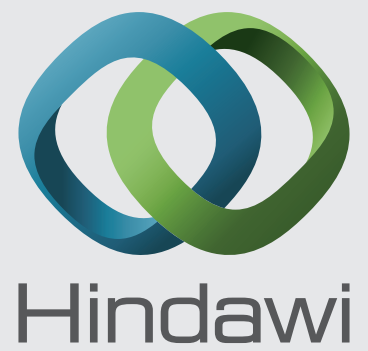

Submit your manuscripts at http://www.hindawi.com
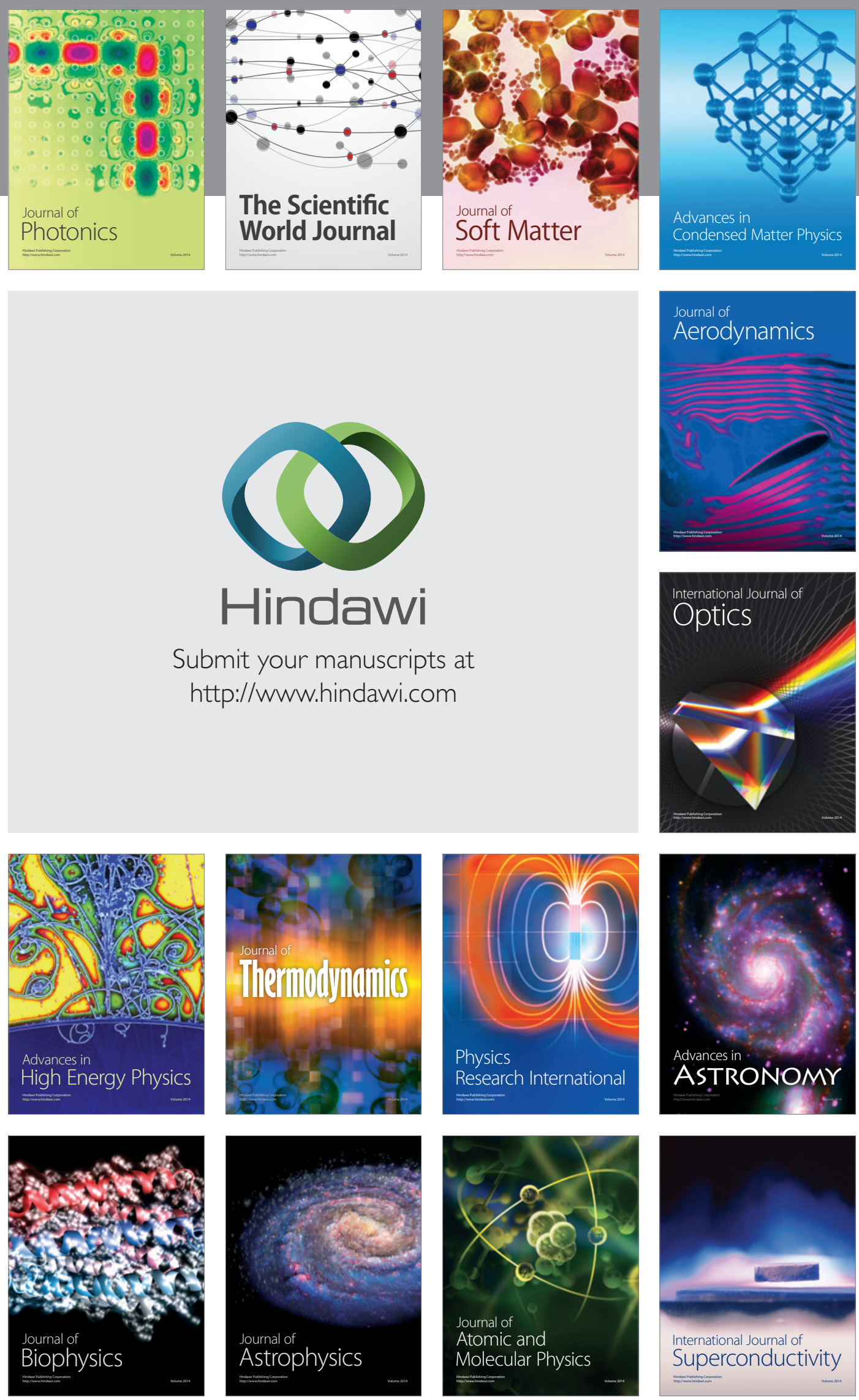
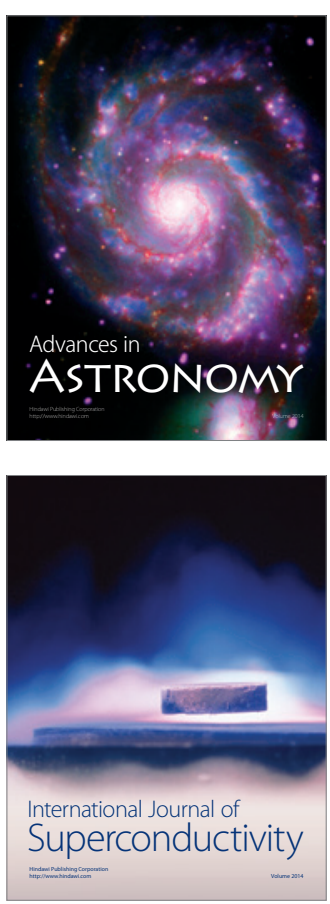